\title{
Identification of Handwritten Complex Mathematical Equations
}

\author{
Sagar Shinde \\ Research Scholar, PAHER University, Udaipur, India \\ Email: sagar.shinde5736@gmail.com \\ Ritu Khanna \\ Professsor, Faculty of Engineering, PAHER University,Udaipur, India \\ Rajendra Waghulade \\ Principal, DNCVP's SMC College, North Maharashtra University, Udaipur, India \\ Received: 05 March 2019; Accepted: 25 March 2019; Published: 08 June 2019
}

\begin{abstract}
The mathematical notation is well known and used throughout the world. Humanity has evolved from simple methods to represent accounts to the current formal notation capable of modeling complex problems. In addition, mathematical equations are a universal language in the scientific world, and many resources such as science and engineering technology, medical field also not an exception containing mathematics have been created during the last decades. However, to efficiently access all that information, scientific documents must be digitized or produced directly in electronic formats.

Although most people are able to understand and produce mathematical information, introducing mathematical equations into electronic devices requires learning special notations or using editors. The proposed methodology is focused on developing a method to recognize intricate handwritten mathematical equations. For pre-processing, Gray conversion and Weiner filtering are used. Segmentation is performed using the morphological operations, which increase the efficiency of the subsequent image of equation. Finally Neural Network based template matching technique is used to recognize the image of handwritten mathematical equation.
\end{abstract}

Index Terms - Neural network, morphological segmentation, recognition, complex equations, template matching.

\section{INTRODUCTION}

Writing in its various forms, printed and handwritten has always been an essential tool in human communication, and also omnipresent in the majority of sectors of its activities. It is used to preserve and archive knowledge. Because of this, man has always developed techniques aimed at its perpetuity throughout the generations. Indeed, with the emergence of new information technologies: electronics and computing, and the new increase in the power of machines, the automation of processing (reading, research and archiving) which are attached to it appears inescapable. It is of concern to researchers in the field of form recognition including automatic recognition of writing. This is how research in this field began over the last few decades.

The automatic recognition of writing is a complex computer process that aims to translate printed or handwritten text into a digitally encoded text, thus understandable by a machine, by transmitting to it the ability to read. The recognition of writing concerns more precisely all tasks related to the mass processing of paper documents. It therefore covers large repetitive applications with large databases such as automatic mathematics equations' recognition, processing of administrative files, automatic sorting of postal mail, reading of amounts and bank checks, processing of postal addresses, Processing of forms, keyboard less interfaces, analysis of written gestures, reading of legacy documents, indexing of library archives and searching for information in databases.

The automatic readings of mathematical equations have seen considerable progress especially in the last decade. This is due, on the one hand, to the many works carried out leading to a variety of different approaches and, on the other hand, to the performance of computers and current acquisition systems coupled with modern statistical methods for example hidden markov models, support vector machines and neural networks. In proposed research methodology, feed forward Backpropagation neural network (BPNN) with scaled conjugate gradient and random data division has been used. The recognition rate has been determined using confusion matrix and precision, accuracy and sensitivity also been calculated.

\section{RELATED WORK}

Optical character recognition is one of the most established thoughts in the automated pattern recognition. In recent time, recognition of mathematical equations turns into the field of useful utilization. With equation's character acknowledgment, the procedure begins with the 
perusing of a scanned picture of a progression of handwritten equation, decides their meaning, and lastly makes an interpretation of the picture to a computer written equation. Likewise, different organizations and persons can utilize this technique to rapidly make an interpretation of mathematical paper reports to computer written documents.

Alvaro et al. (2013) presented a set of hybrid features that combine both on-line and off-line information. Lately, recurrent neural networks have demonstrated to obtain good results and they have outperformed hidden Markov models in several sequence learning tasks, including handwritten text recognition. Hence, this paper also presented a state-of-the-art recurrent neural network classifier and compared its performance with a classifier based on hidden Markov models. Experiments using a large public database showed that both the new proposed features and recurrent neural network classifier improved significantly the classification results [1]. Chaturvedi et al (2014) presented a paper in which a Feed Forward Neural Network and an Izhikevich neuron model is applied for pattern recognition of Digits and Special characters. Given a set of input patterns of digits and Special characters each input pattern is transformed into an input signal. Then the Feed Forward Neural Network and Izhikevich neuron model is stimulated and firing rates are computed. After adjusting the synaptic weights and the threshold values of the neural model, input patterns will generate almost the same firing rate and will recognize the patterns. At last, a comparison between a feedforward neural network which is Artificial Neural Network model and the Izhikevich neural model which is Spiking Neural Network model is implemented in MATLAB for the handwritten pattern recognition [2]. Le et al. (2014) presented a system for recognizing online handwritten mathematical expressions (MEs) and improvement of structure analysis. It represent MEs in Context Free Grammars (CFGs) and employ the CockeYounger-Kasami (CYK) algorithm to parse 2D structure of on-line handwritten MEs and select the best interpretation in terms of symbol segmentation, recognition and structure analysis. This paper proposed a method to learn structural relations from training patterns without any heuristic decisions by using two SVM models. It employs stroke order to reduce the complexity of the parsing algorithm [3]. Liu et al. (2015) presented a method of mathematical formula identification in English PDF document images, which includes three steps: judging columns, extracting mathematical formula character blocks, merging mathematical formula character blocks. Through analyzing and concluding characteristics of the document images in PDF files as well as its effects on mathematical formula identification, this paper designs a related parameter adjustment algorithm for avoiding influences on the performance of mathematical formula identification caused by the resolution variation. The experimental result shows that the adaptability of mathematical formula identification algorithm is improved by some applications [4]. Nguyen et al. (2015) presented application of deep learning to recognize online handwritten mathematical symbols. Recently various deep learning architectures such as Convolution neural network (CNN), Deep neural network (DNN) and Long short term memory (LSTM) RNN have been applied to fields such as computer vision, speech recognition and natural language processing where they have been shown to produce state-of-the-art results on various tasks. This paperapply max-out-based CNN and BLSTM to image patterns created from online patterns and to the original online patterns, respectively and combine them. It also compare them with traditional recognition methods which are MRF and MQDF by carrying out some experiments on CROHME database [5] Khatri et al. (2015) presented a paper which is based on supervised learning vector quantization neural network categorized under artificial neural network. The images of digits are recognized, trained and tested. After the network is created digits are trained using training dataset vectors and testing is applied to the images of digits which are isolated to each other by segmenting the image and resizing the digit image accordingly for better accuracy [6]. Katiyar et al. (2015) presented offline handwritten character recognition of English alphabets using a three layered feed forward neural network. The proposed recognition system describes the evaluation of feed forward neural network by combining four different feature extraction approaches (box approach, diagonal distance approach, mean and gradient operations). The proposed recognition system performs well on the benchmark dataset CEDAR (Centre of Excellence for Document Analysis and Recognition) [7]. Katiyar et al. (2015) proposed an efficient Support Vector Machine based off-line handwritten character recognition system. Experiments have been performed using well known standard database acquired from CEDAR, also it proposes four different techniques of feature extraction to construct the final feature vector. Experimental results show that the performance of SVM is much better than other techniques reported in literature[8]. Chajri et al. (2016) utilized a handwritten mathematical symbols database which is characterized by the diversity of symbols writings in order to have a mathematical symbols recognition system robust and be able to know most of these symbols. This paper used four descriptors (GIST, PHOG, SURF and Centrist) and two classifiers (Artificial Neural Network (ANN) and Support Vector Machines (SVM)) for handwritten mathematical symbols recognition to achieve a comparative study based on the recognition rate [9]. Chajri et al. (2016) presented a new system able to deal with these difficulties and capable to recognize in an efficient manner these expressions. This system is based on four necessary steps: The first concerns the preprocessing techniques (Normalization, Filtering, Binarization and Skew detection and correction). The second and third steps are respectively: the expressions segmentation (connected component algorithm) and the features extraction (Radon transform). The last step is the symbols classification (SVM). This paper also presented the results obtained by using this 
system (expressions segmentation, symbols recognition and the expressions recognition) [10].

\section{SYSTEM ARCHITECTURE AND RESEARCH METHODOLGY}

The system architecture for the recognition of handwritten mathematical equations have been described by using fig. 1 as shown above. The complete flow of preprocessing, segmentation and recognition has been explained.

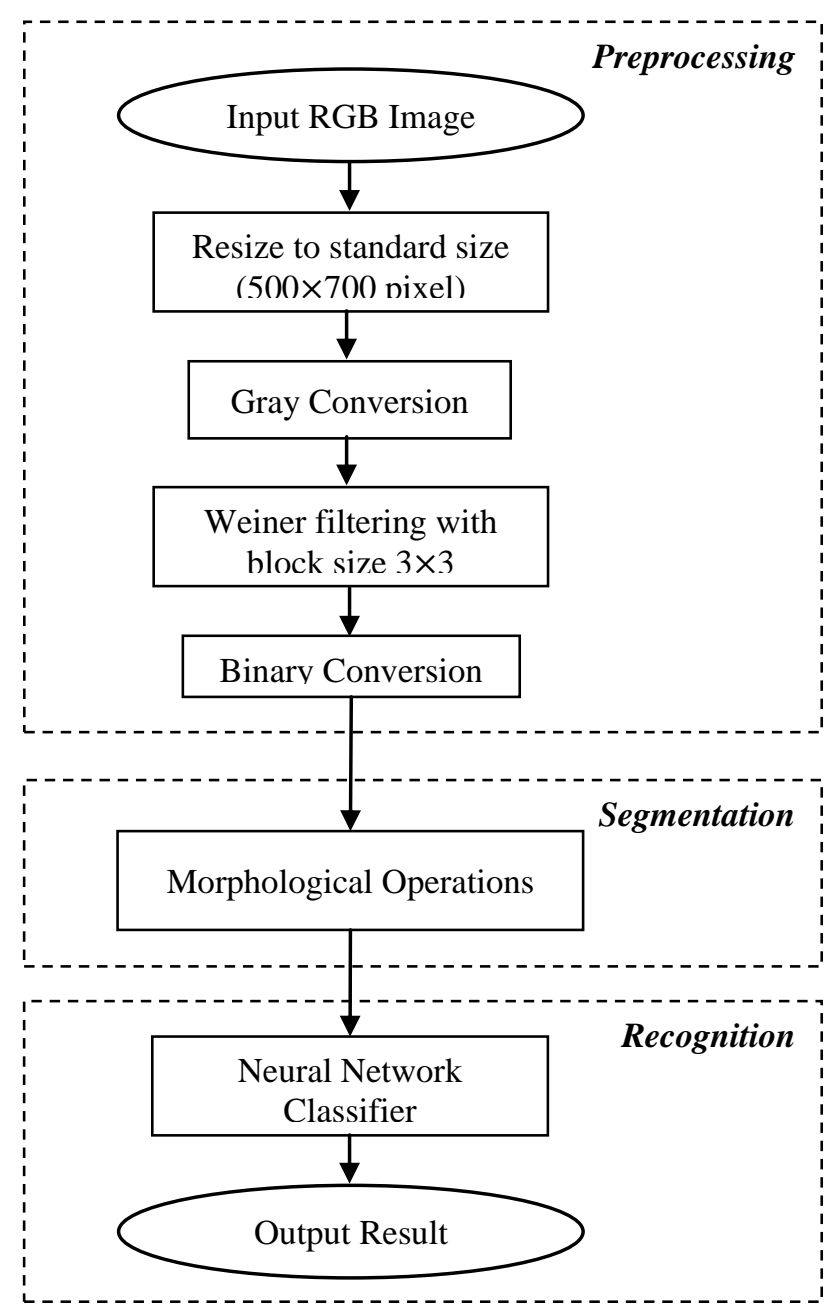

Fig.1. System Architecture for Recognition of Mathematical Equations.

\section{A. Preprocessing}

Generally, the scanned input image contains some irrelevant information or impurities such as holes, dirt particles and the background which must be removed. Actually the input scanned images are affected by various noises. The noise is removed using filtering. The unrelated and surplus part in the background of scanned input image has been removed.

\section{B. Segmentation}

Segmentation is part of the data reduction stage and involves the partitioning of the image plane into meaningful parts, such that "a correspondence is known to exist between images on the one hand and parts of the object on the other hand [11]. In binary morphology, everything could be defined using the set operations. Formally, let I and B are the sets corresponding to the image and structuring element, then

$$
I=\left\{(x, y) \mid I[x, y]<>0, \forall x, y \in I_{R}\right\}
$$

Where $I_{R}$ is the set of all possible (row, column) elements over and image I. B can be defined in a similar manner. There are two basic operations in morphology, which are called dilation and erosion. The dilation of I by $\mathrm{B}$ is denoted by $\mathrm{I} \oplus \mathrm{B}$ and it is defined as

$$
\mathrm{I} \oplus \mathrm{B}=\{\mathrm{c} \mid \mathrm{c}=\mathrm{i}+\mathrm{b}, \text { where } \mathrm{i} \in \mathrm{I}, \mathrm{b} \in \mathrm{B}\}
$$

In the mathematics literature, the dilation is also called as Minkowski addition to refer the inventor of the operator. To complete the dilation operation, B should be translated to the every image pixel and the union of the result should be taken as an overall result. Therefore, to be precise, translation of a set should be defined to shift the structuring element to a specific image point.

The translation of set $\mathrm{B}$ by $\mathrm{t}$ is defined as follows:

$$
\mathrm{B}_{\mathrm{t}}=\{\mathrm{c} \mid \mathrm{c}=\mathrm{b}+\mathrm{t}, \forall \mathrm{b} \in \mathrm{B}\}
$$

Dilation operation is defined as follows:

$$
\operatorname{Dil}(I, B)=\bigcup_{t \in I} I \oplus B_{t}
$$

In the same sense, erosion and erosion operations are defined as in below equations.

$$
\begin{aligned}
& \mathrm{I} \Theta \mathrm{B}=\{\mathrm{c} \mid \mathrm{c}=\mathrm{i}-\mathrm{b}, \mathrm{i} \in \mathrm{I}, \mathrm{b} \in \mathrm{B}\} \\
& \operatorname{Ero}(I, B)=\bigcap_{t \in I} I \Theta B_{t}
\end{aligned}
$$

By using the primitive operations, several morphological operations can be defined. The two basic compound functions that could be constructed by using dilation and erosion are opening and closing respectively. The opening could be defined as dilating an image after eroding. The closing could be defined as eroding and image after dilating. As overall processes it is a smoothing operation that removes the small parasitic areas and smoothes the object contours. The opening of a set I by structuring element B is defined as:

$$
\mathrm{I} \circ \mathrm{B}=(\mathrm{I} \ominus \mathrm{B}) \oplus \mathrm{B}
$$

Similarly, the closing of a set I by structuring element $\mathrm{B}$ is defined as:

$$
\mathrm{I} \cdot \mathrm{B}=(\mathrm{I} \oplus \mathrm{B}) \ominus \mathrm{B}
$$


Finally isolate objects in image reading line wise (left to right in each line). Resize each object to $150 \times 100$

\section{Recognition using Neural Network Classifier}

Artificial neural networks allowed us to formally simulate the work of the human brain, scientists have almost discovered how the human brain's work in terms of scalability and portability of learning memory and the ability to distinguish objects and the ability to make decisions and as we know, the brain is made up of billions of neurons interconnected in a very complex way by neuronal cells, forming an enormous network of neurons associated with them to each other. This correlation between nerve cells gives them the ability to store and deliver information, images, audio and signal sequences that receive across different neurons, neural networks also allow learning through repetition and error.

Learning MLP networks: The learning of a multilayer $\mathrm{NN}$ is generally done by the back-propagation approach, which is the most widely used supervised learning example for MLPs. It uses an optimization method. Universal is to find the network coefficients (weight) that minimize the global error function.

The gradient backpropagation is a method for calculating the error-gradient for every neuron in the network, from the last to the first layer, the principle of back propagation can be described in three basic steps:

Forward Propagation: Neural learning based on this algorithm uses examples of behavior. Assume the learning-base consist of $\mathrm{N}$ learning examples, with each comprised of $\mathrm{x}(\mathrm{n})$ vector applicable to the network inputs, and the vector $d(n)$ of the corresponding desired values for the outputs, the vector $y(n)$ represents the network output for the input $x(n)$. The neural network is supposed to have ' $r$ ' number of output neurons. In this phase, after the initialization of the weights $(w)$, we calculate the input value of each neuron $\mathrm{j}$ of the network which is equal to the weighted sum of the output values of the neurons of the preceding layer, the formula for calculating this value is written as,

$$
\mathrm{VE}_{\mathrm{j}}=\sum_{\mathrm{i} \in \operatorname{pred}(\mathrm{j})} \mathrm{W}_{\mathrm{ij}} \mathrm{VA}_{\mathrm{i}}
$$

The output value (activation) of each neuron $j\left(V_{j}\right)$ is then calculated using transfer function $\mathrm{f}, \mathrm{VA}_{\mathrm{j}}=\left(\mathrm{VE}_{\mathrm{j}}\right)$.

If we use sigmoid function as transfer function $\mathrm{f}$ in our work, the activation value is given by:

$$
V A_{j}=f\left(V E_{j}\right)=\frac{1}{1-e^{-V E_{j}}}
$$

This operation is repeated until the activation values of the output neurons are calculated.

The difference between these values and the corresponding desired values for the outputs represents the learning error called delta: $(\Delta)=d(n)-y(n)$ which must be less than a previously fixed threshold.

Back Propagation: After calculating the learning error which is the result of step of forward propagation of the inputs, in this phase will back-propagate this error through the network layers going from the outputs to the inputs (towards the back), this error will thus be distributed to the neurons of hidden layers so as to be able to adjust in the next phase the weights of the network, the computation of the error $(\Delta)$ of each neuron $\mathrm{j}$ of the hidden layer is made using the formula as shown below.

$$
\Delta_{\mathrm{j}}=\mathrm{VA}_{\mathrm{j}}\left(1-\mathrm{VA}_{\mathrm{j}}\right) \sum_{\mathrm{k} \in \operatorname{succ}(\mathrm{j})} \mathrm{W}_{\mathrm{j}} \mathrm{K} \Delta_{\mathrm{k}}
$$

The Update of the Weights: At the end of the previous step, the learning error was distributed (Back Propagation) to all the neurons of the hidden layers, and now in the current phase we recalculate the weights of the network which are previously initialized with random values using the following rule:

New weight $=$ old weight + learning rate $*$ current neuron error * output of the previous layer. This is described by the formula:

$$
\mathrm{W}_{\mathrm{ij}}=\mathrm{W}_{\mathrm{ij}}+\alpha * \Delta_{\mathrm{j}} * \mathrm{VA}_{\mathrm{i}}
$$

Where, $\alpha$ is the learning rate, which is usually in the range $[0,1]$ (chosen by the user).During the learning of the neural network, these three phases (forward propagation, back-propagation and updating of weights) are repeated as many times as the number of examples of the learning base. Once completed, the mean squared error (MSE) is computed, this measure is often applicable in binary classification where the classes are 0 and 1 . This is a measure related to probabilities; it is defined by the formula:

$$
\text { MSE }=\sqrt{\frac{1}{\mathrm{n}} \sum_{\mathrm{k}=1}^{\mathrm{n}}\left(\mathrm{d}_{\mathrm{k}}-\mathrm{y}_{\mathrm{k}}\right)^{2}}
$$

This is a network validation step where,

$\mathrm{d}_{\mathrm{k}}$ : The desired value in the learning example.

$\mathrm{y}_{\mathrm{k}}$ : The neuron value of the output layer calculated by the MLP.

The obtained MSE value must be below a certain threshold for which the model obtained can be said to respond well to the learning examples; otherwise the procedure will be repeated with other initial values of the $\mathrm{W}_{\mathrm{ij}}$ weights and the learning rate $\alpha$.

The learning phase of a neuron network can therefore be summarized by the algorithm as below.

- Initialization of weights with random values included in a chosen interval

- Reading learning examples.

- Normalize the training data;

- Repeat

- For each learning example D Propagation of the entrance to the front

- Propagation of the error towards the back (backpropagation)

- Update weights 
- End for Computation of the MSE

- As long as the MSE is greater than the threshold or maximum number of iterations.

\section{Simulation Results}

The performance of proposed algorithms has been studied by means of MATLAB simulation.

$$
\begin{array}{cc}
\text { Input RGB Image } & \text { Gray Conversion } \\
x=\frac{-b \pm \sqrt{b^{2}-4 a c}}{2 a} & x=\frac{-b \pm \sqrt{b^{2}-4 a c}}{2 a}
\end{array}
$$

Adaptive Wiener Filtered

$x=\frac{-b \pm \sqrt{b^{2}-4 a c}}{2 a}$

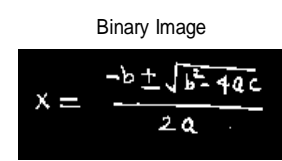

Input RGB Image

$a x^{2}+b x+c=0$

Adaptive Wiener Filtered
$a x^{2}+b x+c=0$

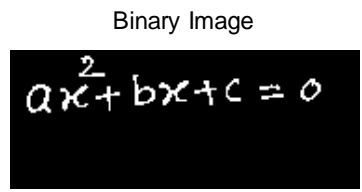

Fig.2. RGB to Gray conversion, filtering and binarization operations on input image-1

Fig. 2 shows the preprocessing steps for the proposed approach. There are four figures named as; Input RGB Image, Gray Conversion, Adaptive Wiener Filtered and Binary Image, show the respective operation on the input image.

The next operation has been explained in fig. 3 where, the required part of equation is extracted through object detection, cropping and mask of equation. Fig.4 shows the output of neural network based matching approach. It shows the matched template for the input equation image. In proposed neural network based approach there is not any threshold value for matching. Neural Network itself does the similarity measure and recognizes test image. Finally, confusion matrix plot shows the performance of proposed method.

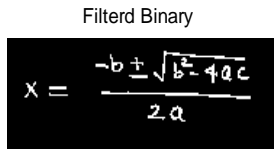

Object Detection

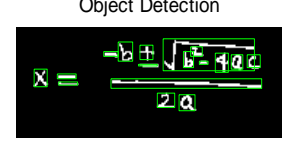

Filterd Binary

$a x^{2}+b x+c=0$
Object Detection

$a x^{2}+b x+c=0$
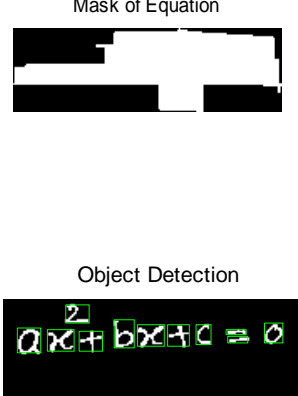

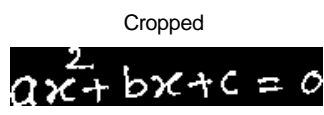

Fig.3. Operation of the second phase i.e. segmentation using morphological operations.

$$
\begin{aligned}
& x=\frac{-b \pm \sqrt{b^{2}-4 a c}}{2 a} \\
& a x^{2}+b x+c=0
\end{aligned}
$$

Fig.4. Matched template with input image-1

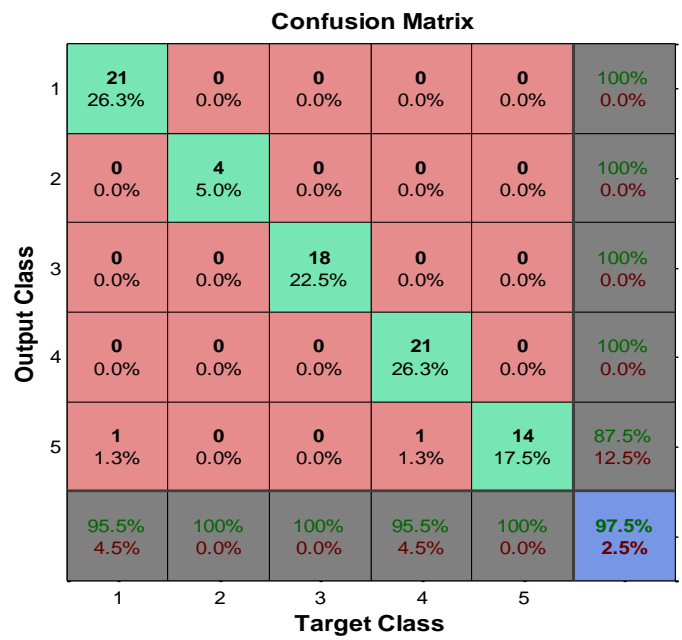

Fig.5. Performance evaluation through confusion matrix 
The row and column are the class of handwritten mathematical equation image database. There are 5 set of classes and each class having different set of detection. For training purpose, it takes 8 images from every class and thus total 40 test images are used. The overall accuracy achieved by proposed approach is $97.5 \%$.

$$
\begin{gathered}
\text { Accuracy }=\frac{T P+T N}{T P+T N+F P+F N} \\
\text { Precision }=\frac{T P}{T P+F P} \\
\text { Sensitivity }=\frac{T P}{T P+F N}
\end{gathered}
$$

Where, $T P=$ True Positive, $T N=$ True Negative, $F P=$ False Positive and $F N=$ False Negative.

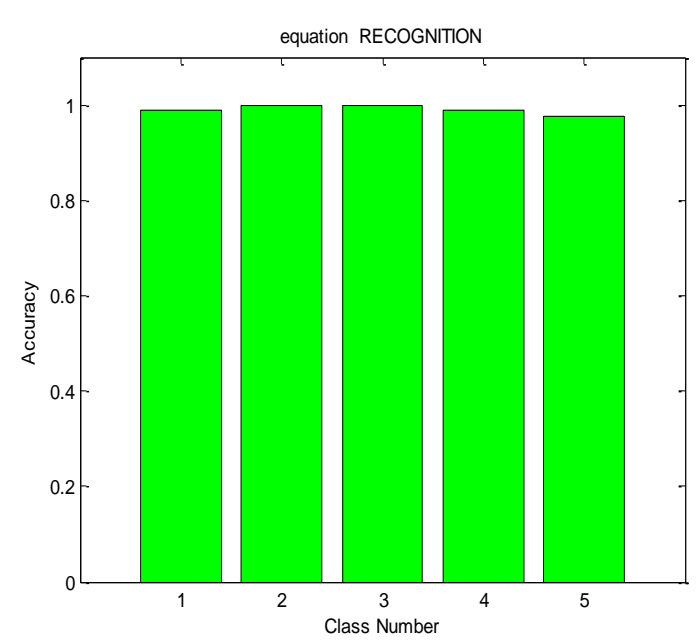

Fig.6. Shows the bar graph of accuracy for handwritten mathematical equation recognition.

The accuracy in recognition has been described using above bar graph as shown in fig. 6 in which the recognition rate of each class number of handwritten mathematical equations have shown in fig. 6. The associated accuracy belongs to various class of equations have shown in percentage as below in Table 1 .

Table 1. Accuracy for handwritten mathematical equation recognition

\begin{tabular}{|c|c|}
\hline Class Number & Accuracy \\
\hline 1 & 0.988235294117647 \\
\hline 2 & 1 \\
\hline 3 & 1 \\
\hline 4 & 0.988235294117647 \\
\hline 5 & 0.975000000000000 \\
\hline
\end{tabular}

Fig.7 shows the bar graph of precision for handwritten mathematical equation recognition. The performance of the system can be determined by using accuracy, precision as well. The associated precision in terms of percentage for this bar graph is shown in Table 2.

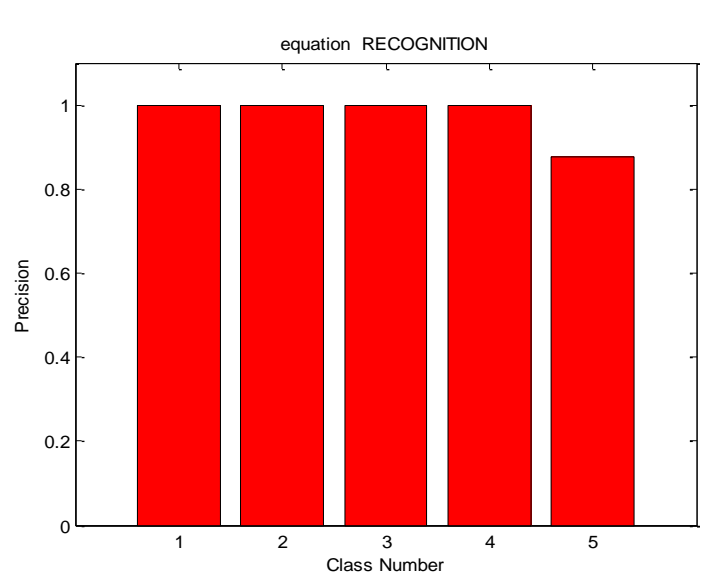

Fig.7. Precision graph for handwritten mathematical equation recognition

Table 2. Precision for handwritten mathematical equation recognition

\begin{tabular}{|c|c|}
\hline Class Number & Precision \\
\hline 1 & 1 \\
\hline 2 & 1 \\
\hline 3 & 1 \\
\hline 4 & 1 \\
\hline 5 & 0.875000000000000 \\
\hline
\end{tabular}

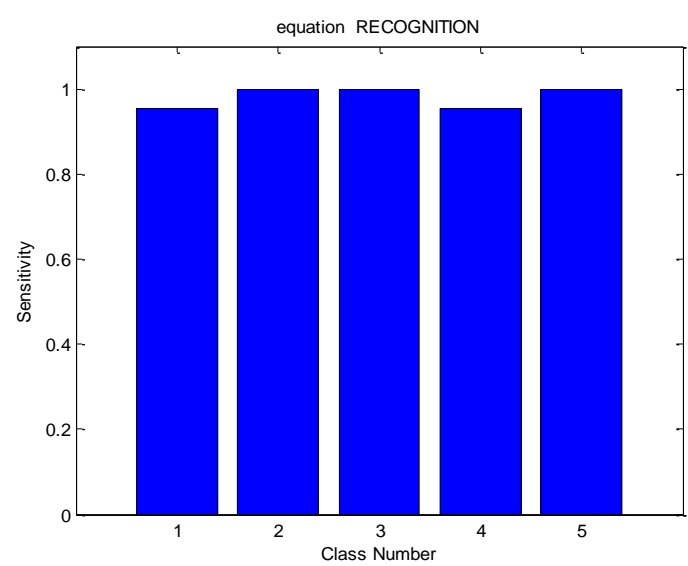

Fig.8. Sensitivity graph for handwritten mathematical equation recognition

Fig. 8 shows the bar graph of sensitivity for handwritten mathematical equation recognition. The sensitivity is main parameter to determine the efficiency as well as evaluation of the overall system. The associated sensitivity in terms of percentage for this bar graph is shown in Table 3.

Table 3. Sensitivity for handwritten mathematical equation recognition

\begin{tabular}{|c|c|}
\hline Class Number & Sensitivity \\
\hline 1 & 0.954545454545455 \\
\hline 2 & 1 \\
\hline 3 & 1 \\
\hline 4 & 0.954545454545455 \\
\hline 5 & 1 \\
\hline
\end{tabular}




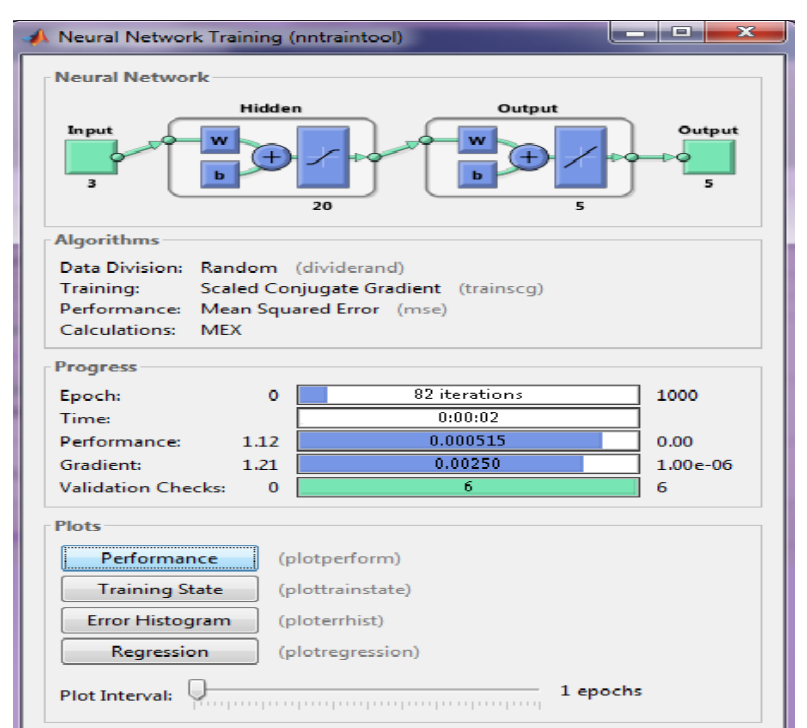

Fig.9. Neural network training

Table 4. Neural Network Parameters

\begin{tabular}{|c|l|l|}
\hline Sl. No. & Parameter & Value/ Type \\
\hline 1 & $\begin{array}{l}\text { Network Training } \\
\text { Function }\end{array}$ & Scale Conjugate Gradient \\
\hline 2 & $\begin{array}{l}\text { Network performance } \\
\text { Function }\end{array}$ & $\begin{array}{l}\text { Mean Squared Error } \\
\text { MSE ) }\end{array}$ \\
\hline 3 & Training & R: 0.99809 \\
\hline 4 & Number of Epochs & 1000 \\
\hline 5 & Number of Iteration & 82 \\
\hline 6 & Performance & 0.0037906 at Epoch 76 \\
\hline 7 & Gradient & 0.0024953 at Epoch 82 \\
\hline 8 & Calculations & MEX \\
\hline
\end{tabular}

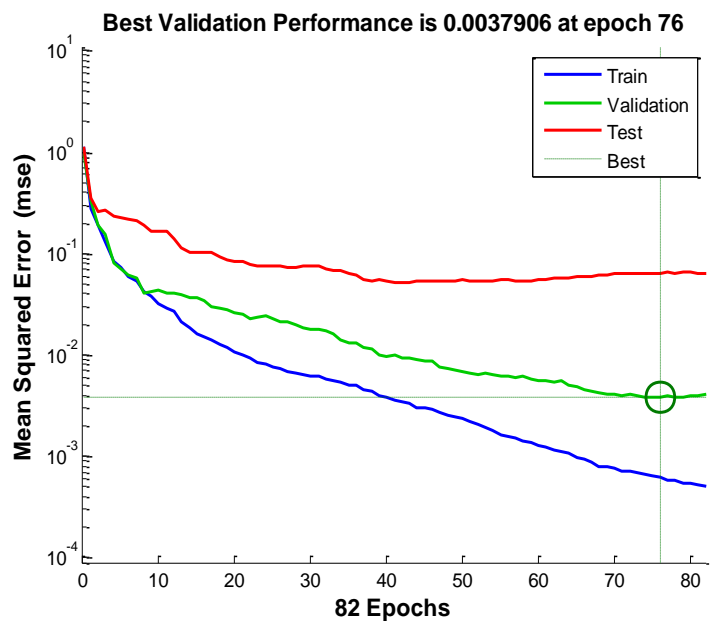

Fig.10. Neural network training performance
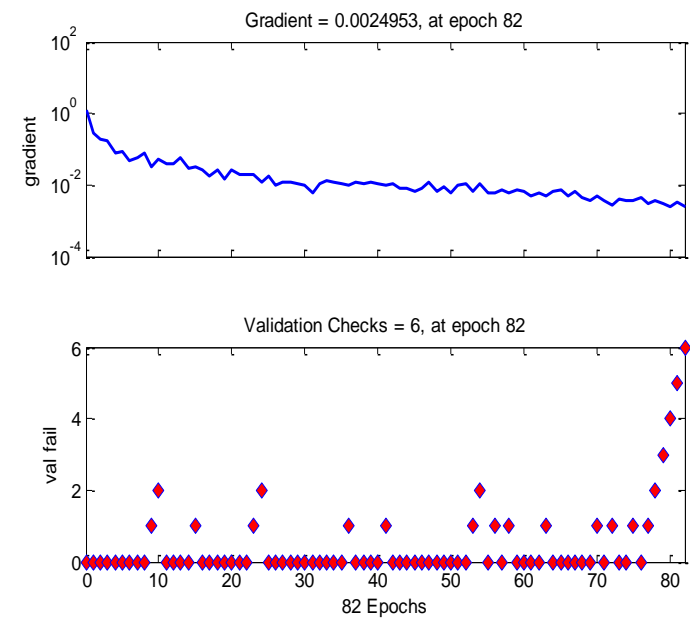

Fig.11. Neural network training state

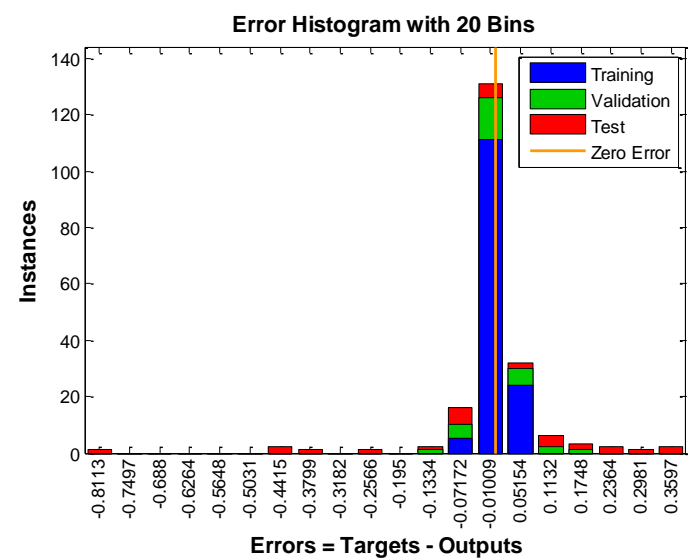

Fig.12. Neural network training error histogram
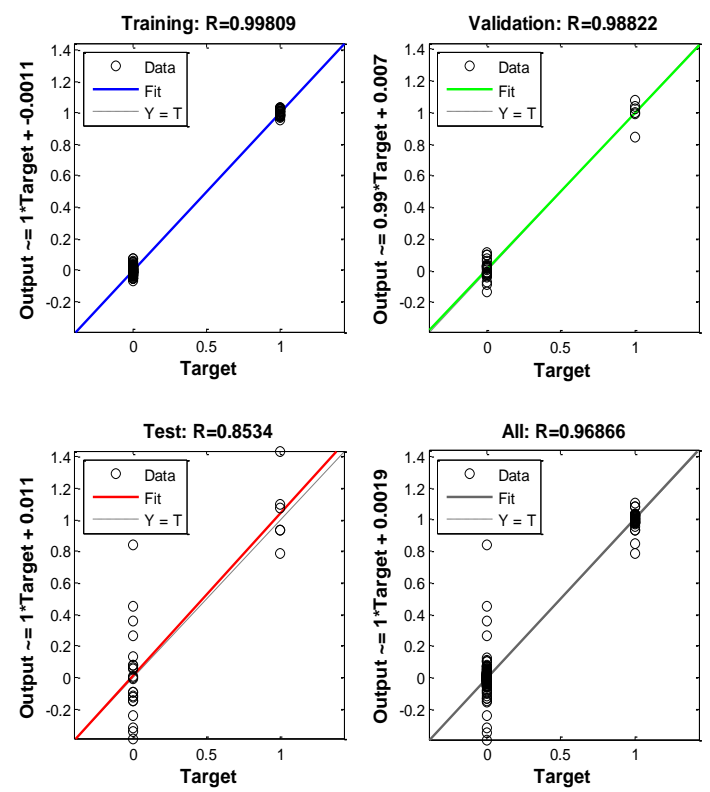

Fig.13. Neural network regression 
The neural network is formed with three input nodes, twenty hidden nodes and three output nodes. The data division is random and network has trained with scaled conjugate gradient and the performance of the network has measured using mean squared error as shown in fig. 9. The performance and efficiency of the neural network can also be observed by using training performance, training state, error histogram through error by subtracting output from target and network regression as shown in fig. 10, fig.11, fig. 12, and fig, 13 respectively.

\section{CONCLUSIONS}

This paper addressed the steps necessary to build a system of handwritten mathematical equation recognition based on morphological operations and neural network matching with scale conjugate gradient for training the network. Simulation results show that the proposed approach successfully recognized the mathematical equation from the given database. In preprocessing approach RGB to gray conversion is used along with resizing. Weiner filtering is used to remove the noise in the image. Main contribution is the study of the implementation of the morphological operation for the segmentation process and finally the neural network base matching provides the recognition accuracy of $97.5 \%$. The performance of the classifier has measured using the error histogram and confusion matrix based on accuracy, precision and sensitivity.

\section{REFERENCES}

[1] Álvaro, Francisco, Joan-Andreu Sánchez, and José-Miguel Benedí. "Classification of on-line mathematical symbols with hybrid features and recurrent neural networks." In Document analysis and recognition (icdar), 2013 12th international conference on, pp. 1012-1016. IEEE, 2013.

[2] Chaturvedi, Soni, Rutika N. Titre, and NehaSondhiya. "Review of handwritten pattern recognition of digits and special characters using feed forward neural network and Izhikevich neural model." In Electronic Systems, Signal Processing and Computing Technologies (ICESC), 2014 International Conference on, pp. 425-428. IEEE, 2014.

[3] Le, AnhDuc, Truyen Van Phan, and Masaki Nakagawa. "A system for recognizing online handwritten mathematical expressions and improvement of structure analysis." In Document Analysis Systems (DAS), 2014 11th IAPR International Workshop on, pp. 51-55. IEEE, 2014.

[4] Liu, Chen, Lina Zuo, Xinfu Li, and Xuedong Tian. "An improved algorithm for Identifying Mathematical formulas in the images of PDF documents." In Progress in Informatics and Computing (PIC), 2015 IEEE International Conference on, pp. 252-256. IEEE, 2015.

[5] Dai Nguyen, Hai, AnhDuc Le, and Masaki Nakagawa. "Deep neural networks for recognizing online handwritten mathematical symbols." In Pattern Recognition (ACPR), 2015 3rd IAPR Asian Conference on, pp. 121-125. IEEE, 2015.

[6] Khatri, Sunil Kumar, Shivali Dutta, and PrashantJohri. "Recognizing images of handwritten digits using learning vector quantization artificial neural network." In Reliability, Infocom Technologies and Optimization
(ICRITO)(Trends and Future Directions), 2015 4th International Conference on, pp. 1-4. IEEE, 2015.

[7] Katiyar, Gauri, and ShabanaMehfuz. "MLPNN based handwritten character recognition using combined feature extraction." In Computing, Communication \& Automation (ICCCA), 2015 International Conference on, pp. 11551159. IEEE, 2015.

[8] Katiyar, Gauri, and ShabanaMehfuz. "SVM based off-line handwritten digit recognition." In India Conference (INDICON), 2015 Annual IEEE, pp. 1-5. IEEE, 2015.

[9] Chajri, Yassine, AbdelkrimMaarir, and BelaidBouikhalene. "A comparative study of handwritten mathematical symbols recognition." In Computer Graphics, Imaging and Visualization (CGiV), 2016 13th International Conference on, pp. 448-451. IEEE, 2016.

[10] Chajri, Yassine, and BelaidBouikhalene. "Handwritten Mathematical Expressions Recognition." International Journal of Signal Processing, Image Processing and Pattern Recognition 9, no. 5 (2016): 69-76.

[11] Ferdinand van der Heijden, "Image Based Measurement Systems, Object Recognition and Parameter Estimation", John Wiley \&Sons, West Sussex, England, 1995.

[12] Gardner, Matt W., and S. R. Dorling. "Artificial neural networks (the multilayer perceptron) - a review of applications in the atmospheric sciences." Atmospheric environment 32, no. 14-15 (1998): 2627-2636.

[13] Plamondon, Réjean, and Sargur N. Srihari. "Online and off-line handwriting recognition: a comprehensive survey." IEEE Transactions on pattern analysis and machine intelligence 22, no. 1 (2000): 63-84.

[14] Mohamed Cheriet, Mounim A. El-Yacoubi, Hiromichi Fujisawa, Daniel P. Lopresti, and Guy Lorette, "Handwriting recognition research: Twenty years of achievementand beyond," Pattern Recognition, vol. 42, pp. 3131-3135, 2009.

[15] Viard-Gaudin, Christian, Pierre-Michel Lallican, and Stefan Knerr. "Recognition-directed recovering of temporal information from handwriting images." Pattern Recognition Letters 26, no. 16 (2005): 2537-2548.

[16] Chen, Qing. "Evaluation of OCR algorithms for images with different spatial resolutions and noises." PhD diss., University of Ottawa (Canada), 2004.

[17] Rhee, TaikHeon, and Jin Hyung Kim. "Efficient search strategy in structural analysis for handwritten mathematical expression recognition." Pattern Recognition 42, no. 12 (2009): 3192-3201.

[18] Wang, Xin, Guangshun Shi, and Jufeng Yang. "The understanding and structure analyzing for online handwritten chemical formulas." In Document Analysis and Recognition, 2009. ICDAR'09. 10th International Conference on, pp. 1056-1060. IEEE, 2009.

[19] Yuan, Zhenming, Hong Pan, and Liang Zhang. "A novel pen-based flowchart recognition system for programming teaching." In Advances in Blended Learning, pp. 55-64. Springer, Berlin, Heidelberg, 2008.

[20] Feng, Guihuan, Christian Viard-Gaudin, and Zhengxing Sun. "On-line hand-drawn electric circuit diagram recognition using 2D dynamic programming." Pattern Recognition 42, no. 12 (2009): 3215-3223.

[21] Szwoch, Mariusz. "Guido: a musical score recognition system." In Document Analysis and Recognition, 2007. ICDAR 2007. Ninth International Conference on, vol. 2, pp. 809-813. IEEE, 2007.

[22] Çelik, Mehmet, and BerrinYanikoğlu. "Handwritten mathematical formula recognition using a statistical approach." In Signal Processing and Communications 
[23] Applications (SIU), 2011 IEEE 19th Conference on, pp. 498-501. IEEE, 2011.

\section{Authors' Profiles}

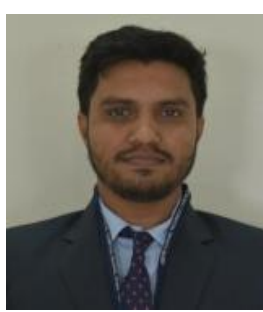

Sagar Shinde is currently working as an Assistant Professor in JSPM, Narhe Technical Campus, Pune. He received his B. E (E \& TC) from from DYPIET, SPPU, Pune and M. E (E \& TC) from North Maharashtra University, Jalgaon. $\mathrm{He}$ is a Research Scholar in Electronics \& communication engineering at PAHER, Udaipur. His three patents have been published. One on real time gas leak detection \& controlling, second on energy efficient railway tunnel \& third on handwritten mathematical equations recognition. His Area of Interest is image processing and neural network.

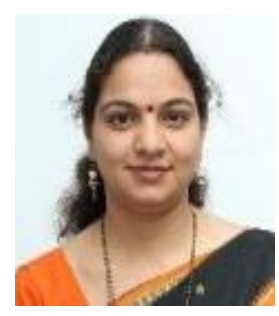

Prof. Dr. Ritu Khanna is currently working as a professor in faculty of engineering of pacific academy of higher education and research university, Udaipur. She has published more than 20 research papers in national and international journals.

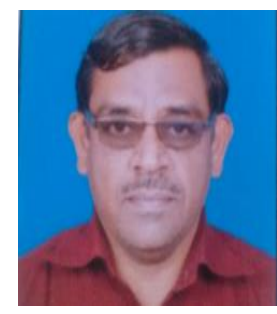

Prof. Dr. R. B. Waghulade is currently working as a Principal in DNCVP, North Maharashtra University, Jalgaon. $\mathrm{He}$ received his bachelor degree in Electronics from Pune University in 1989 and Master Degree in Electronics in 1991. He received his $\mathrm{PhD}$ from North Maharashtra University in year 2008. He has published 17 books on computer and electronics field and got funding from North Maharashtra University for two research projects. He has published no. of research papers and one patent on handwritten mathematical equations recognition.. His area of interest is in Wireless Sensors and Image Processing.

How to cite this paper: Sagar Shinde, Ritu Khanna, Rajendra Waghulade, "Identification of Handwritten Complex Mathematical Equations", International Journal of Image, Graphics and Signal Processing(IJIGSP), Vol.11, No.6, pp. 45-53, 2019.DOI: 10.5815 /ijigsp.2019.06.06 